in systemic sclerosis. Rheumatol Int 2012;32:3581-6.

2. Eckardt J, Towfigh H. Hypercalcinosis of the hands in scleroderma: 2 case reports. Handchir Mikrochir Plast Chir 1994;26:330-4.

3. Merlino G, Germano S, Carlucci S. Surgical management of digital calcinosis in CREST syndrome. Aesthetic Plast Surg 2013;37:1214-9.

4. Lapner MA, Goetz TJ. High-speed burr debulking of digital calcinosis cutis in scleroderma patients. J Hand Surg Am 2014;39:503-10.

\section{Wrist Pain due to Abnormal Extensor Carpi Ulnaris Tendon}

SuRak Eo ${ }^{1}$, Sujin Bahk ${ }^{1}$, Neil F. Jones ${ }^{2}$

${ }^{1}$ Department of Plastic and Reconstructive Surgery, Dongguk University Graduate School of Medicine, Seoul, Korea; ${ }^{2}$ Center for Hand and Upper Extremity Surgery, University of California Irvine, Irvine, CA, USA

Correspondence: Neil F. Jones

Center for Hand and Upper Extremity Surgery, University of California Irvine, 101 The City Drive South, Pavillion III, Building 29A, Orange, California 92868, USA Tel: +1-714-456-5759, Fax; +1-714-456-7547, E-mail: nfjones@uci.edu

No potential conflict of interest relevant to this article was reported.

Received: 27 Jul 2015 • Revised: 11 Nov $2015 \bullet$ Accepted: 28 Dec 2015 pISSN: 2234-6163 • elSSN: 2234-6171

http://dx.doi.org/10.5999/aps.2016.43.4.389

Arch Plast Surg 2016;43:389-390

Copyright (C) 2016 The Korean Society of Plastic and Reconstructive Surgeon This is an Open Access article distributed under the terms of the Creative Commons Attribution Non-Commercial License (http://creativecommons.org/licenses/by-nc/4.0) which permits unrestricted non-commercial use, distribution, and reproduction in any medium, provided the original work is properly cited.

Pain arising from lesions in the extensor carpi ulnaris (ECU) is known to occur mainly due to tenosynovitis, tendon rupture, and subluxation or dislocation of the ECU tendon $[1,2]$. However, anomalies of the ECU tendon itself and the surrounding extensor retinaculum are also possible factors contributing to the impairment of function at the dorso-ulnar aspect of the wrist and little finger [2,3]. We report a case in which an abnormal ECU tendon slip was revealed during exploration of the wrist.

A 28-year-old right-handed female neurosurgeon visited our clinic due to dorso-ulnar wrist pain in her dominant hand for six months. The pain was exacerbated during wrist motion, especially during forearm supination for handling the needle holders in the operating field. A physical examination revealed no sensory changes and no specific signs, including the Phalen and Finkelstein tests. Radiographic studies also showed no problems such as joint subluxation. The hand showed a slight tender swelling of the ECU tendon around the wrist. With the suspicion of tenosynovitis, steroid injection into the sixth compartment was performed. However, the pain did not respond to medical treatment. Electrodiagnostic studies revealed no abnormalities. On exploration, we found the abnormal insertion of an additional ECU tendon slip into the radial inner side of the sixth compartment (Fig. 1). After simple excision of the entire abnormal ECU tendon slip, her symptoms improved.

The ECU tendon is enclosed in the sixth compartment of the extensor retinaculum and various anomalies of the ECU tendon have been reported $[2,3]$. The prevalence of an accessory tendinous slip arising from the ECU tendon has been reported to be $10 \%$ to $34 \%[2,4]$, and Barfred and Adamsen [3] noted that the accessory tendinous slip of the ECU was sometimes connected with the extensor digiti minimi. Nakashima classified accessory slips into three types according to the insertion point of the main tendon to the fifth metacarpal bone: type $\mathrm{A}$, on the base along with the main tendon; type $\mathrm{B}$, on the midsection; and type $\mathrm{C}$, on the head [2]. The case presented here belonged to type A. The average width of the slips has been reported to range from $1-1.53 \mathrm{~mm}[2,3]$, while the

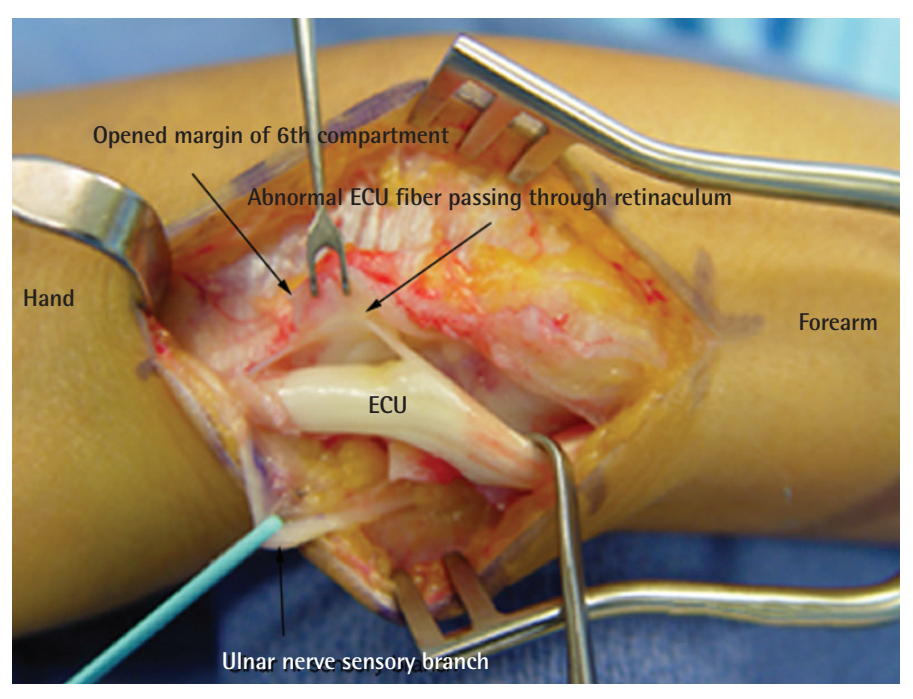

Fig. 1.

The abnormal insertion of an additional extensor carpi ulnaris (ECU) tendon slip into the radial inner side of the sixth compartment. 
presented case showed a slip $1.5 \mathrm{~mm}$ in width and 2 $\mathrm{cm}$ in length. Taleisnik et al. [5] observed that the transverse fibers of the medial wall of the sixth compartment extend proximally to become confluent with the epimysium of the ECU, and are reinforced by the linea jugata, which normally prevents ECU tendon subluxation during full supination. Since the ECU tendon is exposed to maximal traction when the forearm is supinated, we believe that her wrist pain was induced by repetitive and excessive supination-pronation activity, which might otherwise remain non-symptomatic given usual movement patterns. Anatomical variations of the ECU may affect diagnostic and surgical procedures involving the dorso-ulnar aspect of the wrist. During surgery performed on the ECU, the surgeon must be aware of the possible existence of an accessory tendinous slip that may cause ECU lesions to persist.

\section{References}

1. Chun S, Palmer AK. Chronic ulnar wrist pain secondary to partial rupture of the extensor carpi ulnaris tendon. J Hand Surg Am 1987;12:1032-5.

2. Nakashima T. An accessory extensor digiti minimi arising from extensor carpi ulnaris. J Anat 1993;182(Pt 1):109-12.

3. Barfred T, Adamsen S. Duplication of the extensor carpi ulnaris tendon. J Hand Surg Am 1986;11:423-5.

4. Mestdagh H, Bailleul JP, Vilette B, et al. Organization of the extensor complex of the digits. Anat Clin 1985;7:4953.

5. Taleisnik J, Gelberman RH, Miller BW, et al. The extensor retinaculum of the wrist. J Hand Surg Am 1984;9:495-501. 